\title{
Frequency Domain Extended-MUSIC Algorithm for TOA Estimation in Indoor UWB Radio Impulse Channels
}

H. Meknessi

F. Harabi
A. Gharsallah

\begin{abstract}
Several indoor localization systems have grown considerably, in order to obtain a high performance in dense multipath propagation channels. Many studies have been done in this area. Different methods have been proposed based on the use of Impulse Radio Ultra Wide band (IR-UWB) signals. These systems are usually based on time of arrival estimation using different algorithms. In this paper, a new time of arrival estimation algorithm is proposed for impulse radio UWB systems, which is based on the EXTENDED-MUSIC algorithm. Furthermore, Simulation results show these performances in multipath channels. The proposed method is compared with other high resolution algorithms such as Multiple SIGNAL Classification (MUSIC), estimation of signal parameters via rotational invariance technique (ESPRIT) and the MATRIX PENCIL method. Simulation will justify the high accuracy and resolution localization capabilities of the proposed algorithm.
\end{abstract}

\section{Keywords}

Impulse Radio Ultra Wide band (IR-UWB), Time of arrival estimation (TOA), EXTENDED-MUSIC, ESPRIT, MATRIX PENCIL

\section{INTRODUCTION}

One of most promising application areas of wireless location systems for indoor positioning is the Ultra wideband (UWB) radio signals. The first research on this field appeared in 1960 by Ross and Robbins [1] these researches titled impulse system. In 1986 the American defence department used the term of Ultra Wide band (UWB) for these systems.

UWB has become the focus of developments more recently for both wireless data communication and real time location tracking. It was the subject of recent standardisation efforts both in the USA in 2002 and the European Union in 2007 [2].

The IR-UWB has become the more interesting technology for wireless communication applications such as the indoor localization. Therefore, those performances in multipath channels have been discussed in many studies [3-4].

UWB signals employ the transmission of very short impulses of radio energy whose characteristic spectrum signature extends across a wide range of radio frequencies [5]. Since it has many advantages to penetrate obstacles and reduce interference in dense multipath environments with high accuracy, also, the ability of pulsed UWB to resolve individual multi-path components [6-7].

The IR-UWB systems send DS-UWB using binary phase pulse amplitude modulation in dense multipath propagation channels (PAM). With very large bandwidths, IR-UWB has extremely accurate location estimates and provides high frequency-domain resolution.

Moreover, the time of arrival is the most popular parameter in the wireless geo-localization systems on determining the time propagation signal from the transmitter to the receiver; it is characterized by the high accuracy in the indoor environment with simple positioning algorithms.

On account of the high time resolution nature, UWB positioning based on TOA estimation methods becomes a superior alternative [8].

In the literature performances of many super-resolution techniques for TOA estimation are evaluated [9-10], [11-12] and [13-14]. In this paper, we proposed a frequency-domain time-of-arrival (TOA) estimation method based on the Extended MUSIC algorithm [15] for indoor localization applications. The method will be compared with the best known methods such as MUSIC, ESPRIT and MATRIX PENCIL algorithms in terms of accuracy and resolution.

The rest of the paper is organized as follows: Section 2 presents the indoor UWB channel model. Section 3 elaborate the proposed FREQUENCY EXTENDED MUSIC method and develop the MUSIC, Esprit and MATRIX PENCIL algorithms in frequency domain. Simulation results are provided in Section 4 and section 5 concludes the paper.

These superscripts: (.)T, (.)*, (.)H and (.)-1 presents the matrix transpose, conjugate, hermitian, and inverse operators, respectively

\section{MULTIPATH INDOOR UWB RADIO CHANNEL}

The model of UWB signal for binary phase pulse amplitude modulation (PAM) is given by

$$
s(t)=\sum_{j=-\infty}^{+\infty} \sum_{n=0}^{N_{c}} f_{j} c_{n} p\left(t-j T_{s}-n T_{c}\right)
$$

Where $f_{j} \in\{-1,+1\}$ is the biphase data symbols and $c_{n} \in$ $\{1,+1\}$ is the user-specific code sequence of period $N_{c} \cdot p(t)$ is the waveform of the transmitted pulse, which is the second order derivative of Gaussian function given by

$$
p(t)=\left(1-4 \pi t^{2} / \tau_{m}^{2} \exp \left(-2 \pi t^{2} / \tau_{m}^{2}\right)\right)
$$

$T_{c}$ is the chip duration and $T_{s}$ is defined as the symbol duration. Therefore, the most channels propagation model used in UWB systems is the Saleh-Valenzuela model [16]. In this model, the impulse response is presented as a period composed of $\mathrm{K}$ clusters and $\mathrm{L}$ paths in each cluster.

The UWB multipath channel impulse model in the $\mathrm{k}^{\text {th }}$ cluster defined as

$$
h^{(k)}(t)=\sum_{i=1}^{L} \alpha_{i}^{(k)} \delta\left(t-\tau_{i}\right)
$$

Where $\alpha_{i}^{(k)}$ is the fading coefficient and $\tau_{i}$ is the propagation delay of $1^{\text {th }}$ path relative to the $\mathrm{k}^{\text {th }}$ cluster. 
For UWB systems, the received wideband signal can be represented as the convolution of $s(t)$ and $h^{(k)}(t)$ as:

$x^{(k)}(t)=s(t) * h^{(k)}(t)+b^{(k)}(t)$

Then the received wideband signal can be formulated as follow:

$$
x^{(k)}(t)=\sum_{i=1}^{L} \alpha_{i}^{(k)} s\left(t-\tau_{i}\right)+b^{(k)}(t)
$$

Where $b^{(k)}$ is the additive Gaussian white noise in the $\mathrm{k}^{\text {th }}$ cluster.

After Fourier transformation to the received signal in (5), can be formulated as follow:

$$
X^{(k)}(f)=S^{k}(f) \sum_{i=1}^{L} \alpha_{i} e^{-j 2 \pi f \pi i}+B^{k}(f)
$$

Where $X^{(k)}(f), \quad S^{k}(f)$ and $\quad B^{k}(f)$ are the Fourier transformation of $x^{(k)}(t), s(t)$ and $b^{(k)}(t)$ respectively.

Then sampling (6) $\mathrm{N}$ frequencies uniformly over a given UWB frequency bandwidth $B$. The spaced frequency sampling interval is $\Delta \mathrm{f}=\mathrm{B} / \mathrm{N}$. In order to avoid inter symbol interference in the time domain, $\Delta \mathrm{f}$ is determined to satisfy the condition of $1 / \Delta f \geq 2 \tau \max$, where $\tau \max$ is the maximum delay of the measured UWB channel.

The measurement data in the $\mathrm{k}^{\text {th }}$ cluster is written as:

$$
X^{(k)}(f)=S^{k}(f) \sum_{i=1}^{L} \alpha_{i} e^{-j 2 \pi n \Delta f i i}+B^{k}(f)
$$

\section{Where $\mathrm{n}=0,1,2 \ldots \mathrm{N}$}

The received signals in the frequency domain can then be given in matrix from as:

$$
X=A S+B
$$

Where

$$
\begin{aligned}
& X=[X(1), X(2), \ldots X(k)] \\
& S=[S(1), S(2), \ldots S(k)] \\
& A=\left[a\left(\tau_{1}\right), a\left(\tau_{2}\right), \ldots a\left(\tau_{L}\right)\right] \\
& B=[B(1), B(2), \ldots B(k)]
\end{aligned}
$$

Where:

$$
\begin{aligned}
& X(k)=\left[X_{0}(k), X_{1}(k), \ldots X_{N-1}(k)\right]^{T} \\
& B(k)=\left[B^{k}(0), B^{k}(1), \ldots B^{k}(N-1)\right]^{T} \\
& a\left(\tau_{l}\right)=\left[1, e^{-j 2 \pi n \Delta f \tau_{i}}, \ldots, e^{-j 2 \pi(N-1) \Delta f \tau_{L}}\right]^{T} \\
& S(k)=\left[S(n) \alpha_{1}^{(k)}, S(n) \alpha_{2}{ }^{(k)}, \ldots, S(n) \alpha_{L}{ }^{(k)}\right]^{T}
\end{aligned}
$$

Finally the covariance matrix is expressed as:

$$
R=E\left[X X^{T}\right]
$$

Let the number of samples $\mathrm{N}$ be larger than the number of paths L. Then, from the formulation of received signals and additive noise is obtained as:

$$
R=A S_{E} A^{T}+I \sigma^{2}
$$

Where the L*L vector $S_{E}=E\left[S S^{T}\right]$ is the source covariance matrix, and the $\mathrm{N} * \mathrm{~N}$ vector I is the identity matrix, and $\boldsymbol{\sigma}^{2}$ is the noise variance.

\section{TOA ESTIMATION ALGORITHMS}

In order to obtain high localization accuracy in indoor environment, many algorithms have been developed for time delay estimation [17-18] and [19-20].

\subsection{MUSIC method}

MUSIC is the abbreviation of Multiple Signal Classification; the MUSIC algorithm was developed by Schmidt in 1979. It is the one of the high resolution subspace TOA algorithms. It based on the property of orthogonality of the signal subspace and the noise subspace, this method is based on the Eigenvalues decomposition of the correlation matrix.

The following steps summarize the MUSIC algorithm:

1. Perform Eigenvector and Eigenvalue on R. Where $e_{i}$ is the eigenvector associated with Eigenvalue $\lambda_{i}$ which will organized as :

$\lambda_{1} \succ \lambda_{2} \succ \ldots \lambda_{L} \succ \lambda_{L+1}=\lambda_{L+2} \ldots=\lambda_{N}=\sigma_{w}^{2}$

2. Let $\mathrm{U}_{\mathrm{s}}=\left[\mathrm{e}_{1} \ldots \mathrm{e}_{\mathrm{L}}\right]$ and $\mathrm{U}_{\mathrm{n}}=\left[\mathrm{e}_{\mathrm{L}+1} \ldots \mathrm{e}_{\mathrm{N}}\right]$ be the signal subspace and noise subspace, respectively.

3. Construction of the MUSIC pseudospectrum function as :

$$
F(\tau)=1 / a(\tau){ }^{H} U_{n} U_{n}^{H} a(\tau)
$$

Where $a(\tau)$ is the steering vector.

4. Find the values of $\tau$ that minimizes $F(\tau)$, these values are the estimated TOA.

\subsection{ESPRIT method}

ESPRIT stands for estimation of Signal Parameters via rotational Invariance Technique. It developed by ROY and KEILATH[21]. This subspace based method explores the rotational invariance property in the subspace signal created by two sub-arrays derived from original array with a translation invariance structure.

The ESPRIT algorithm is summarized as follows:

1. Perform Eigen values decomposition on R.

2. Extraction of the subspace signal.

3. Decompose the subspace into 2 subarray $E$ and $\mathrm{E}_{2}$.

4. Estimate the matrix $\Psi$ using the method of least squares

$$
\Psi=\left(E_{1}{ }^{H} E_{1}\right)^{-1} E_{1}^{H} E_{2}
$$

5. Calculate the Eigen values of $\Psi$ 
6. The TOA is obtained from Eigenvectors of $\Psi$

\subsection{MATRIX PENCIL method}

The Matrix PENCIL technique is based on the use of the received signal expression. It was developed in order to estimate the poles of a system [22].

The Pencil Matrix algorithm is summarized as follows:

1. Choose the Pencil parameter $\mathrm{P}$ as $\frac{L}{3} \leq P \leq \frac{2 * L}{3}$

2. Construction of the received signals matrix.

3. Decompose the received signals matrix into 2 subarrays $\mathrm{X}_{0}$ and $\mathrm{X}_{1}$ as :

$$
X_{0}=\left[\begin{array}{cccc}
x(0) & x(1) & \ldots & x(P-1) \\
x(1) & x(2) & \ldots & x(P) \\
\cdot & \cdot & & . \\
\cdot & \cdot & . & \\
x(L-P-1) & x(L-P) & \ldots & x(L-2)
\end{array}\right]
$$

and

$$
X_{1}=\left[\begin{array}{cccc}
x(1) & x(2) & \ldots & x(P) \\
x(2) & x(3) & \ldots & x(P+1) \\
\cdot & \cdot & & \cdot \\
\cdot & \cdot & \cdot & \cdot \\
x(L-P) & x(L-P+1) & \ldots & x(L-1)
\end{array}\right]
$$

4.

$$
\text { Estimate the matrix } \Psi_{M P} \text { using the method of least }
$$$$
\text { square } \Psi_{M P}=\left(X_{0}{ }^{H} X_{0}\right)^{-1} X_{0}^{H} X_{1}
$$

5. The TOA is obtained from Eigenvectors of $\Psi_{M P}$

\subsection{FREQUENCY EXTENDED-MUSIC} Method

FREQUENCY EXTENDED-MUSIC method is used to estimate TOA of IR-UWB in a multipath communication channel which is based on Extended MUSIC algorithm [15]. The following steps outline the procedure of estimation of the TOA using the proposed method:

1. Perform Eigenvalues decomposition on $\mathrm{R}$

$$
\lambda_{n}, \mathrm{n}=1, \ldots, \mathrm{N}
$$

2. Calculate a positive scalar value as $m=\frac{\operatorname{tr}(\hat{R})}{N}$

3. Construct the new Frequency EXTENDED MUSIC function as:

$$
G^{\tau}=R+m a(\tau) a(\tau)^{H}
$$

4. Extract the Eigenvalues of the new extended correlation matrix $G^{\tau}$ in decreasing order

$$
\begin{aligned}
& \mu_{l} \geq \lambda_{l} \quad l=1, \ldots, L \\
& \mu_{n}=\lambda_{n}=\sigma_{n}^{2} \quad n=L+1, \ldots, N
\end{aligned}
$$

5. Calculate the function

$$
D(\tau)=\frac{1}{\sum_{l=L+1}^{N}\left(\mu_{l}-\lambda_{l}\right)}
$$

\section{SIMULATION RESULTS}

In this section, we investigate and compare the performances of our proposed approach with high resolution algorithms such as MUSIC, ESPRIT and PENCIL MATRIX method. Two main evaluations are considered: the resolution and the precision. Uniform linear array has been used in all simulations and the simulation parameters are given in Table1.

Table 1: Simulation Parameters

\begin{tabular}{|c|c|}
\hline Parameters & Value \\
\hline Measurements & 50 \\
\hline DS-UWB signals duration & $2 \mathrm{~ns}$ \\
\hline UWB pulse duration & $1 \mathrm{~ns}$ \\
\hline Frequency sampling interval & $1.3 \mathrm{GHz}$ \\
\hline $\begin{array}{c}\text { Sampling frequencies number } \\
(\mathrm{N})\end{array}$ & 200 \\
\hline
\end{tabular}

In the first simulation, two rays of UWB arriving signals with a signal to noise ratio (SNR) of $0 \mathrm{~dB}$ and delays of $0.6 \mathrm{~ns}$ and $0.62 \mathrm{~ns}$, respectively, are considered. The illustrated results, with a snapshot number of 50, are shown in Fig. 1 and Table 2. The obtained spectrums of MUSIC and Frequency Extended MUSIC are plotted as a function of the TOA in Fig.1. However, table 2 illustrates those of ESPRIT and Pencil Matrix methods.

Table 2: TOA estimation by ESPRIT and PENCIL MATRIX

\begin{tabular}{|c|c|c|c|}
\hline Delays & True Delay & PENCIL MATRIX & ESPRIT \\
\hline Delay 1 & $0.60 \mathrm{~ns}$ & $-0.1564 \mathrm{~ns}$ & $-0.1691 \mathrm{~ns}$ \\
\hline Delay 2 & $0.62 \mathrm{~ns}$ & $0.2222 \mathrm{~ns}$ & $-0.1497 \mathrm{~ns}$ \\
\hline
\end{tabular}



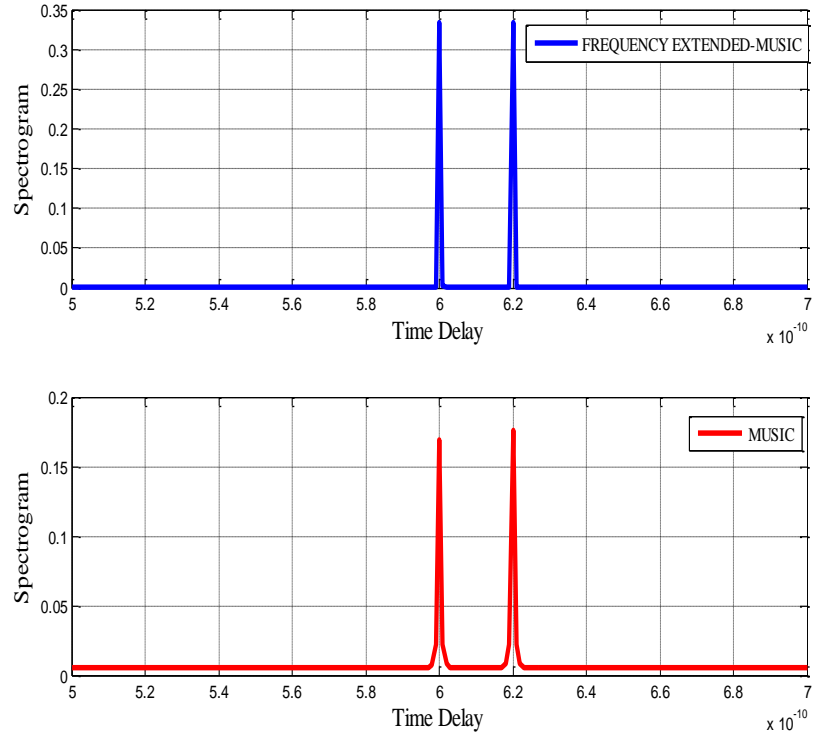

Fig. 1: Frequency Extended-Music And Music spectrograms of tow sources located at $0.6 \mathrm{~ns}$ and $0.62 \mathrm{~ns}$

From the illustrated results, it is clear that our proposed approach and the MUSIC algorithm are able to perform an accurate detection and separation for two neighboring paths scenario. Indeed, both MUSIC and proposed algorithm can prominently distinguish the TOA, contrary to ESPRIT and Pencil Matrix methods which are completely failed to solve TOA estimation of the considered signals in this complicate scenario. This result proves that good performances in terms of resolution can be achieved with the proposed FREQUENCY EXTENDED-MUSIC method.

To study the performance limits of the proposed method on term of precision, it use as a performance parameter the mean square error (MSE) defined as follows:

$$
M S E=1 / n b r \sum_{i=1}^{n b r}(\hat{\tau}-\tau)^{2}
$$

Where $\hat{\tau}$ is the estimated time of arrival for the $\mathrm{i}^{\text {th }}$ measurement.

With respect of the same simulation settings already used in Fig. 1, the MSE of TOA estimation by FREQUENCY EXTENDED-MUSIC and MUSIC algorithms is evaluated for different SNR levels and the obtained results are plotted in Fig. 2. It used for this simulation two rays of UWB arriving signals with delays of $0.04 \mathrm{~ns}$ and $0.35 \mathrm{~ns}$, respectively.

The results obtained on fig. 2 provide the high performances of FREQUENCY EXTENDED-MUSIC method. It can see that, the estimation accuracy of the proposed method increases greatly as soon as the SNR levels increases. For a $\mathrm{SNR}=-10 \mathrm{~dB}$, we reach an estimation error $=0^{\circ}$. However at high SNRs (higher than $-7 \mathrm{~dB}$ ), these two methods have the same performance.

With the same simulation condition The MSE versus the number of snapshots is shown in Fig. 3.

It seems clear that at high number of snapshots the performance of FREQUENCY EXTENDED-MUSIC and MUSIC algorithms is the same, whereas for lower value our proposed method is more accurate. This result is very important because to have good accuracy with a limited number of observations give opportunities to apply the proposed method for real-time applications.

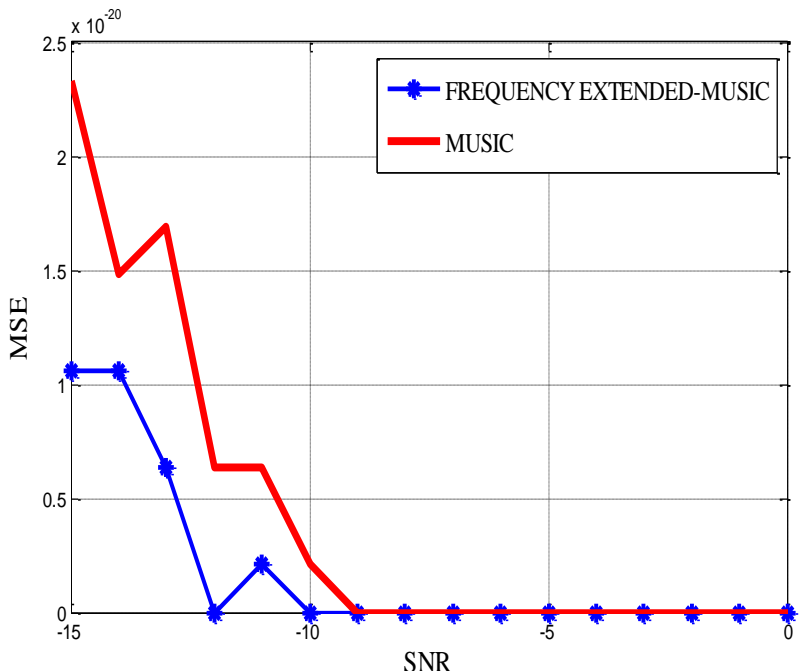

Fig. 2: MSE for tow paths located at $0.04 \mathrm{~ns}$ and $0.35 \mathrm{~ns} u$ versus SNRs

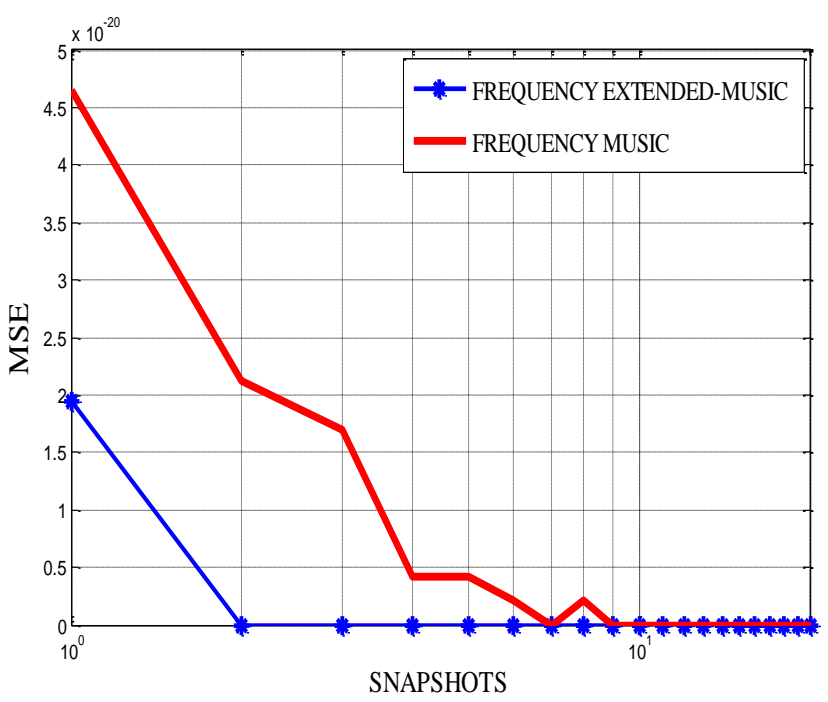

Fig. 3: MSE for tow paths located $0.04 \mathrm{~ns}$ and 0.35 versus snapshots

\section{CONCLUSION}

Based on the EXTENDED MUSIC algorithm, a new approach is proposed in this paper for time of arrival estimation in indoor UWB radio impulse channels. In fact, we combine and adapt for the estimator to IR-UWB Model. Furthermore our proposed method can process the signals in order to estimating their location.

The effectiveness of the proposed algorithm was verified, also, the conducted simulations prove the high performances of our method in a dense multipath environment. Moreover, Indeed the FREQUENCY EXTENDED MUSIC is able not only to estimate time delay multipath at low SNR but also with few snapshot number. Therefore, our proposed approach can ensure fast and robust TOA estimation as compared to the classic MUSIC algorithm. Moreover, In conclusion, the proposed method seems perfect to be applied in ultrawideband localization systems for applications in indoor environment, known as Real Time Location Systems (RTLS), 
where the robustness and the estimation speed are very required.

In future work, we plan to evaluate the performance our proposed approach in a realistic indoor environment, which is the major problem of indoor localization is the presence of Non-line-of-sight (NLOS) channels. We will also to obtain an algorithm with better performances and lower complexity.

\section{REFERENCES}

[1] Ross, G.F., "A Time Domain criterion for the design of wideband radiating elements". IEEE Trans. on Antennas \& Propagation, 16, 355, 1968.

[2] Washington, DC, FCC 02-48, Apr. 2002.

[3] S. Pittet, V. Renaudin, B. Merminod, "UWB and MEMS Based Indoor Navigation" The journal of navigation, vol. 61,no. 3, pp. 369-384, July2008.

[4] G. Morrison, M. Fattouche, "Super- Resolution Modeling of the indoor Radio Propagation Channel" IEEE Transactions on vehicular technology, vol. 47, no. 2, pp. 649-657, May 1998.

[5] Y.-H. You, J. B. Kim, "Pilot and data symbol-Aidek frequency estimation for UWB-OFDM" Progress In Electromagnetics Research, 2009.

[6] M. Z. Win, R. A. Scholtz, "On the robustness of ultra wide bandwidth signals in dense multipath en vironments," IEEE Commun. Lett., vol. 2, no. 2, pp. 5153, Feb. 1998.

[7] W. Zhang, Thushara D. Abhayapala, J. Zhang, "UWB Spatia-Frequency Channel Characterization" Vehicular Technology Conference, vol. 6, pp. 2732 - 2736, 2006.

[8] F. Wang , X. Zhang, " Joint Estimation of TOA and DOA in IR-UWB System using Sparse Representation Framework" ETRI Journal, vol. 36, no. 3, pp. 460-468, June. 2014.

[9] Ch. Wann, Sh.Hsiung Hsu, "Estimation and Analysis of Signal Arrival Time for UWB Systems" Vehicular Technology Conference, vol. 5, pp. 3560 - 3564, 2004.

[10] D. Rui , Q. Zhihong, J. Hong, "TOA estimation for IRUWB system using Matrix Pencil'International Forum on Information Technology and Applications, vol. 1, pp. $461-464,2009$.

[11] D. Mustapha, H. Zaher, Y. Nekachtali, K. Drouiche, "TOA Estimation Technique for IR-UWB Based on HomogeneityTest" ETRI Journal, vol. 35, no. 5, pp. 757-766, Octobr. 2013.
[12] X. Li, K. Pahlavan, and J. Beneat, "Performance of TOA Estimation techniques in indoor multipath channels" PIMRC, vol. 2, pp. 911-915, 2002.

[13] Oh. Daegun, Go. Seungryeol, Ch Jong-wha, “ PacketReduced Ranging Method with Superresolution TOA Estimation Algorithm for Chirp-Based" ETRI Journal , vol. 35, no. 3, pp. 361-370, June. 2013.

[14] Li . Xinyang, J. Hong: "Lunar Rover Positioning Based on TOA Estimation for UWB Signal Using UnitaryESPRIT" The Ninth International Conference on Electronic Measurement \& Instruments ICEMI, pp. 2646 - 2-650, 16-19 Aug. 2009

[15] F. Harabi,H. Changuel, A.Gharsallah, “ A new estimation of direction of arrival a special antenna shape" Smart Mater, Struct.J., pp. 2595-2599, 2007.

[16] Adel A. M. Saleh and Reinaldo A. Valenzuela, "A Statistical Model for Indoor Multipath Propagation", IEEE J. in communications, vol. sac-5, No.2, February 1987

[17] M.Pallas, G.Jourdain, "Active high resolution Time Delay Estimation for large BT signals ", IEEE Trans,SP, vol.39, , 781-788, Apr.1991 .

[18] X. Li and K. Pahlavan, "Super-resolution TOA estimation with diversity for idoor geolocation," IEEE Trans. on Wireless Communications, vol.3, no. 1, pp 224-234, Jan. 2004.

[19] C. Falsi, D. Dardari, L. Mucchi, and M. Z. Win, “Time of arrival estimation for UWB localizers in realistic environments," EURASIPJ. Applied Signal Processing, pp. 1-13, 2006

[20] F. Ge et al., "Super-Resolution Time Delay Estimation in Multipath Environments," IEEE Trans. Circuits Syst. I: Regular Papers, vol. 54, no. 9, Sept. 2007, pp. $1977-$ 1986.

[21] Roy, A. Paulraj and T. Kailath, "Estimation of signal parameters via rotational invariance techniques ESPRIT", Advanced Algorithms and Architectures for Signal Processing, vol. 696, pp. 94-101, 1986.

[22] R. S. Adve, O. M. Pereira-Filho, T. Sarkar, and S. Rao, "Extrapolationof time domain responses from three dimensionalobjects utilizing the matrix pencil technique," IEEE Transactionson Antennas and Propagation, vol. 45, pp. 147-156, January 1997. 